\title{
Cultural Humility in Nursing Practice
}

\author{
Aimable Nkurunziza ${ }^{1}$, Alice Nyirazigama ${ }^{1}$, Isabelle Soulé ${ }^{1}$ \\ ${ }^{1}$ University of Rwanda, College of Medicine and Health Sciences, Byumba School of Nursing and Midwifery, \\ Rwanda
}

\section{Background}

In 2012, Human Resources for Health (HRH) Rwanda brought together international nursing experts with widely varying backgrounds, worldviews, and values. This phenomenon has generated an increased awareness of the impact of culture on attitudes, behaviors, and professional practices.

\section{Description}

Culture is complex and it influences the development of individual beliefs, attitudes, and values. Understanding the influence of culture and its effect on interpretive meaning is essential for healthcare providers in a globalized world and requires intellectual, attitudinal and behavioral flexibility. Broadly speaking, in order to work effectively within an international team, individuals must be interested in other cultures, be sensitive enough to notice cultural differences, and then be willing to modify their attitudes and behavior as an indication of respect for those differences. Yet, integrating a foreign perspective is fundamentally unnatural, and apprehension, resistance, and judgment are common responses.

For example, Rwandan colleagues work from a collectivist viewpoint. Collectivists perceive themselves as part of a group and emphasize interdependence over independence, affiliation over confrontation, and cooperation over competition. In contrast, the U.S. healthcare system is based on individualism, rooted in a belief in the separation and autonomy of individuals. Individualism recognizes the individual, not the group, as the basic unit of survival. Both collectivism and individualism have merits. However, it is important to understand that each relies on different values in decision-making, that behaviors may differ as a result, and that those behaviors may be in conflict.

\section{Conclusions}

Cultural humility, defined as the lifelong commitment to self-critique and developing mutually beneficial partnerships, is needed in order to work effectively across diverse worldviews. In a model of cultural humility, the most serious barrier to culturally appropriate care is the nurses' failure to develop self-awareness and a respectful, open attitude toward diverse points of view. Interacting from a starting point of humility, rather than professional expertise or arrogance, generates a different, more positive, partnership and healthcare encounter.

Key words: culture, cultural humility, partnership, global health, diversity 